\title{
Trajectory Controllability of the Systems Governed by Hilfer Fractional Systems
}

\author{
Vishant Shah*1, Jaita Sharma $^{\dagger 1}$, Prakashkumar H. Patel ${ }^{\ddagger 2}$ and Haribhai R. Kataria $\S 3$ \\ ${ }^{1}$ Department of Applied Mathematics, Faculty of Technology and Engineering, The M. S. University of \\ Baroda, Vadodara - 390 001, India. \\ ${ }^{2}$ Department of Mathematics, Krishna School of Science, Drs. Kiran \& Pallavi Patel Global University, \\ Varnama, Vadodara - 391243, India. \\ ${ }^{3}$ Department of Mathematics, Faculty Science, The M. S. University of Baroda, Vadodara - 390 001, India.
}

\begin{abstract}
In this manuscript, we consider a nonlinear system governed by Hilfer fractional integro-differential equations in a Banach space. Using the concept of operator semigroup and Gronwall's inequality, we have established the trajectory controllability of the integro-differential equation with local and non-local conditions. Finally, we have given an example to illustrate the application of the derived results.
\end{abstract}

Keywords- Hilfer Fractional order differential equation, Trajectory controllability, Gronwall's inequality, $C_{0}$-semigroup

AMS Subject Classification: 26A33, 34GXX, 34G20, 47D06

\section{Introduction}

From the past few decades, the differential equations involving fractional order derivatives are receiving the increasing interest by many researchers due to numerous applications in widespread areas of science and engineering such as in models of epidemiology, medicines, electrical and mechanical engineering, biochemistry etc. For more applications one can refer [1,2]. It has been verified that the fractional differential equations are more accurate to describe the dynamical behavior of a real life phenomenon more precisely. Hilfer [2] proposed new fractional derivative which is a generalization of RiemannLiouville fractional derivative and Caputo fractional derivative. There after many researchers studied the qualitative properties of the solution like existence, uniqueness and stability of fractional differential

*vishantmsu83@gmail.com

†jaita.sharma-appmath@msubaroda.ac.in

¥prakash5881@gmail.com

$\S$ hrkrmaths@yahoo.com 
equations including Hilfer fractional differential operators. The study of qualitative properties are find in the papers [3 8] and monographs [2,9], and reference their in.

The aim of this article is to study the trajectory controllability of the system governed by Hilfer fractional integro-differential system in the infinite dimensional Banach space with classical and nonlocal conditions. Trajectory controllability is finding the control of the system which steers the initial state to desired final state of the system via prescribed trajectory. There are many physical systems in aerodynamics for which we required trajectory controllability of the system for cost effectiveness. Therefore, trajectory controllablity is stronger then any other controllability. Controllability of linear and nonliear systems in finite and infinite dimensional spaces are found in the articles [10 21] and reference their in. Singh [22] studied the exact controllability of Hilfer fractional differential systems.

Study of trajectory controllability of integer order linear and nonlinear systems are found in article 23, 24 and same for fractional order finite and infinite dimensional systems are found in [25 27].

The aim of this article is to study Trajectory controllability of infinite dimensional Hilfer fractional control systems of the form:

$$
\mathcal{D}_{0+}^{\lambda, \mu} u(\zeta)+A u(\zeta)=g\left(\zeta, u(\zeta), \int_{0}^{\zeta} a(\zeta, \tau, u(\tau)) d \tau\right)+w(\zeta)
$$

over the interval $[0, T]$ with classical condition $\mathcal{I}_{0+}^{(1-\lambda)(1-\mu)} u(0)=u_{0}$ and non-local conditions $\mathcal{I}_{0+}^{(1-\lambda)(1-\mu)}[u(0)-$ $h(u)]=u_{0}$.

\section{Preliminaries}

In this section we are going to discuss basic definitions and results to derive controllability conditions.

Definition 2.1. [28] For $\lambda>0$, the fractional integral of order $\lambda$ of a function $h(\zeta)$ is defined by

$$
\mathcal{I}_{\zeta_{0}}^{\lambda} h(\zeta)=\frac{1}{\Gamma(\lambda)} \int_{\zeta_{0}}^{\zeta}(\zeta-\tau)^{\lambda-1} f(\tau) d \tau
$$

provided the integral on right exist.

Definition 2.2. [28] The Hilfer fractional derivative of order $\lambda, 0<\lambda<1$ and type $\mu, 0 \leq \mu \leq 1$ is defined by

$$
\mathcal{D}_{\zeta_{0}+}^{\lambda, \mu} h(\zeta)=\mathcal{I}_{\zeta_{0}+}^{\mu(1-\lambda)} \frac{d}{d \zeta} \mathcal{I}_{\zeta_{0}+}^{(1-\lambda)(1-\mu)} h(\zeta)
$$

provided the right value exist.

Definition 2.3. [28] For all $\theta \in \mathbb{C}$ and $\lambda>0$, the Wright-type function $M_{\lambda}$ is defined as:

$$
M_{\lambda}(\theta)=\sum_{n \in \mathbb{N}} \frac{(-\theta)^{n-1}}{\Gamma(1-\lambda n)(n-1) !}
$$

provided the sum on the right exist. 
The Wright-type function satisfy the following properties:

(1) $M_{\lambda}(\theta)>0$ for all $\lambda>0$.

(2) For $-1<\iota<\infty$ the integral, $\int_{0}^{\infty} \theta^{\eta} M_{\lambda}(\theta) d \theta=\frac{\Gamma(1+\eta)}{\Gamma(1+\lambda \eta)}$

(3) For $r>0$ the integral, $\int_{0}^{\infty} \frac{\lambda}{\theta^{\lambda+1}} e^{-r \theta} M_{\lambda}\left(\theta^{-\lambda}\right) d \theta=e^{-r^{\lambda}}$

Let, $\mathcal{C}_{\mathcal{T}}=C([0, T], X)$, the set of all continuous functions from $[0, T]$ into Banach space $X$ under the norm given by $\|\Psi\|_{T}=\sup _{0 \leq \zeta \leq T}\|\Psi(\zeta)\|$.

Let, $\mathcal{T}(\zeta)$ be the family of semi-group generated by the linear operator $-A$. We define two two linear operators $\mathcal{S}_{\lambda}$ and $\mathcal{Q}_{\lambda}$ as:

$$
\begin{aligned}
\mathcal{S}_{\lambda}(\zeta) & =\int_{0}^{\infty} M_{\lambda}(\theta) \mathcal{T}\left(\zeta^{\lambda} \theta\right) d \theta \\
\mathcal{Q}_{\lambda}(\zeta) & =\int_{0}^{\infty} \lambda \theta M_{\lambda}(\theta) \mathcal{T}\left(\zeta^{\lambda} \theta\right) d \theta
\end{aligned}
$$

We have following result for the operators $\mathcal{S}_{\lambda}$ and $\mathcal{Q}_{\lambda}$.

Lemma 2.1. If $\mathcal{T}(\zeta)$ be the family of $C_{0}$-semigroup generated by the linear operator $-A$ for all $\zeta \in[0, T]$ then the families of operators $\mathcal{S}_{\lambda}(\zeta)$ and $\mathcal{Q}_{\lambda}(\zeta)$ defined by 2.2) and (2.3) are:

(1) continuous and bounded for all $\zeta \in[0, T]$.

(2) strongly continuous over the interval $\zeta \in(0, T]$

Proof. Since, the family $\mathcal{T} \zeta$ is $C_{0}$-semigroup generated by the linear operator $-A$ therefore there exist $M \geq 0$ such that $\|\mathcal{T}(\zeta)\| \leq M$.

For any $u \in X$

$$
\left\|\mathcal{S}_{\lambda}(\zeta) u\right\| \leq \int_{0}^{\infty} M_{\lambda}(\theta)\left\|\mathcal { T } ( \zeta ^ { \lambda } \theta ) \left|\||| u\| d \theta \leq M \int_{0}^{\infty} M_{\lambda}(\theta) d \theta\|u\| \leq M\|u\| \quad\right.\right. \text { (using property-1) }
$$

and

$$
\left\|\mathcal{Q}_{\lambda}(\zeta) u\right\| \leq \int_{0}^{\infty} \lambda \theta M_{\lambda}(\theta)\left\|\mathcal{T}\left(\zeta^{\lambda} \theta\right)\right\|\|u\| d \theta \leq M \int_{0}^{\infty} \lambda \theta M_{\lambda}(\theta)\|u\| d \theta \leq \frac{M}{\Gamma(\lambda)}\|u\| \quad \text { (using property-2) }
$$

Therefore the operators $\mathcal{S}_{\lambda}(\zeta)$ and $\mathcal{Q}_{\lambda}(\zeta)$ are bounded with bound $M$ and $M / \Gamma(\lambda)$ respectively.

Let $\left\{u_{n}\right\}$ be any sequence in a Banach space $X$ converges to $u \in X$ and consider,

$$
\left\|\mathcal{S}_{\lambda}(\zeta) u_{n}-\mathcal{S}_{\lambda}(\zeta) u\right\|=\left\|\mathcal{S}_{\lambda}(\zeta)\left[u_{n}-u\right]\right\| \leq M\left\|u_{n}-u\right\|
$$

Therefore, $\mathcal{S}_{\lambda}(\zeta)$ is continuous. Similarly, we can prove that $\mathcal{Q}_{\lambda}(\zeta)$ is continuous for all $\zeta \in[0, T]$. Clearly, families $\mathcal{S}_{\lambda}(\zeta)$ and $\mathcal{Q}_{\lambda}(\zeta)$ are strongly continuous as

$$
\left\|\mathcal{S}_{\lambda}\left(\zeta_{2}\right) u-\mathcal{S}_{\lambda}\left(\zeta_{1}\right) u\right\| \leq \int_{0}^{\infty} M_{\lambda}(\theta)\left\|\mathcal{T}\left(\zeta_{2}^{\lambda} \theta\right)-\mathcal{T}\left(\zeta_{1}^{\lambda} \theta\right)\right\|\|u\| d \theta \leq\left\|\mathcal{T}\left(\zeta_{2}^{\lambda} \theta\right)-\mathcal{T}\left(\zeta_{1}^{\lambda} \theta\right)\right\|\|u\|
$$

which tends to zero as $\zeta_{2} \rightarrow \zeta_{1}$ for all $0<\zeta_{1}<\zeta_{2} \leq T$ and $u \in X$. Similarly, family $\mathcal{Q}_{\lambda}(\zeta)$ is also strongly continuous. This completes the proof of the lemma. 
With the help of families of the operators we define two linear operators $\mathcal{S}_{\lambda, \mu}(\zeta)$ and $\mathcal{K}_{\lambda}(\zeta)$ as:

$$
\begin{aligned}
\mathcal{S}_{\lambda, \mu}(\zeta) & =\mathcal{I}_{0}^{\mu(1-\lambda)} \mathcal{K}_{\lambda}(\zeta) \\
\mathcal{K}_{\lambda}(\zeta) & =\zeta^{\lambda-1} \mathcal{Q}_{\lambda}
\end{aligned}
$$

The operators satisfies following properties:

Lemma 2.2. If $\mathcal{T}(\zeta)$ be the family of $C_{0}$-semigroup generated by the linear operator $-A$ for all $\zeta \in[0, T]$ then the families of operators $\mathcal{S}_{\lambda, \mu}(\zeta)$ and $\mathcal{K}_{\lambda}(\zeta)$ defined by (2.4) and (2.5) are:

(1) continuous and bounded for all $\zeta \in[0, T]$.

(2) strongly continuous over the interval $\zeta \in(0, T]$

Proof. Clearly,

$$
\left\|\mathcal{K}_{\lambda}(\zeta) u\right\| \leq \zeta^{\lambda-1}\left\|\mathcal{Q}_{\lambda}(\zeta) u\right\| \leq \frac{\zeta^{\lambda-1} M}{\Gamma(\lambda)}\|u\|
$$

and

$$
\left\|\mathcal{S}_{\lambda, \mu}(\zeta) u\right\| \leq \frac{1}{\Gamma(\mu(1-\lambda))} \int_{0}^{\zeta}(\zeta-\tau)^{\mu(1-\lambda)-1} \tau^{\lambda-1} \frac{M}{\Gamma(\lambda)}\|u\| d \tau \leq \frac{M(\mu(1-\lambda)) \zeta^{\lambda+\mu-\lambda \mu-1}}{\Gamma(\lambda+\mu-\lambda \mu-1)}\|u\|
$$

for all $\zeta \in[0, T]$ and $u \in X$. Therefore the operators $\mathcal{S}_{\lambda, \mu}(\zeta)$ and $\mathcal{K}_{\lambda}(\zeta)$ are bounded for $\zeta \in[0, T]$.

The continuity and strong continuity of the operators $\mathcal{S}_{\lambda, \mu}(\zeta)$ and $\mathcal{K}_{\lambda}(\zeta)$ are achieved using Lemma2.1. This completes the proof of the Lemma.

Consider the control system governed by Hilfer fractional integro-differential equation with classical condition in a Banach space $X$ over the interval $[0, T]$

$$
\begin{aligned}
\mathcal{D}_{0+}^{\lambda, \mu} u(\zeta)+A u(\zeta) & =g\left(\zeta, u(\zeta), \int_{0}^{\zeta} a(\zeta, \tau, u(\tau)) d \tau\right)+w(\zeta) \\
\mathcal{I}_{0+}^{(1-\lambda)(1-\mu)} u(0) & =u_{0}
\end{aligned}
$$

where, $\mathcal{D}_{0+}^{\lambda, \mu}$ is Hilfer fractional derivative operator. $A$ is closed linear operator which is infinitesimal generator of $C_{0}$ semigroup and $u_{0} \in X . w \in U$, a Hilbert space.

Definition 2.4. A function $u \in \mathcal{C}_{\mathcal{T}}$ is called mild solution of integro-differential equation (2.6) if $u$ is solution of the integral equation

$$
u(\zeta)=\mathcal{S}_{\lambda, \mu} u_{0}+\int_{0}^{\zeta} \mathcal{K}_{\lambda}(\zeta-\tau)[g(\tau, u(\tau), S u(\tau))+w(\tau)] d \tau
$$

where, $S u(\zeta)=\int_{0}^{\zeta} a(\zeta, \tau, u(\tau)) d \tau$, operators $\mathcal{S}_{\lambda, \mu}$ and $\mathcal{K}_{\lambda}(\zeta)$ are defined as 2.4 and 2.5 respectively.

Definition 2.5. The system 2.6] is said to be completely controllable on the interval $[0, T]$ if for any $u_{0}, u_{1} \in X$ there exit a control $w(\zeta) \in U$ such that the solution 2.7) of 2.6 satisfies $u(T)=u_{1}$. 
Definition 2.6. The system (2.6) is totally controllable on the interval $[0, T]$ if it is completely controllable over all its sub intervals $\left[\tau_{k}, \tau_{k+1}\right]$.

Let $\mathcal{T}$ be the set of all functions $y(\cdot)$ defined over the interval $[0, T]$ satisfying $y(0)=u_{0}$ and $y(T)=u_{1}$ and Hilfer fractional derivative exist everywhere. The set $\mathcal{T}$ is called set of all feasible trajectories.

Definition 2.7. The system $(2.6)$ is is said to be trajectory controllable (T-Controllable) any $y \in \mathcal{T}$, there exist $L^{2}$ control function $w \in U$ such that the solution $u(\zeta)(2.7)$ satisfy $u(\zeta)=y(\zeta)$ almost everywhere.

Trajectory controllability of system is strongest controllability in comparison with other controllability.

\section{Trajectory Controllability with Classical Condition:}

In this section, we are going to discuss trajectory controllability of the system governed by Hilfer fractional integro-differential equation (2.6). To discuss it we make following conditions.

(A1) Linear operator $-A$ is the infinitesimal generator of $C_{0}$-semigroup.

(A2) The nonlinear function $g:[0, T] \times X \times X \rightarrow X$ satisfies

$$
\left\|g\left(\zeta, u_{1}, v_{1}\right)-g\left(\tau, u_{2}, v_{2}\right)\right\| \leq L_{1}(r)\left\|u_{1}-v_{1}\right\|+L_{2}(r)\left\|u_{2}-v_{2}\right\|
$$

for all $\zeta, \tau \in[0, T], u_{1}, u_{2}, v_{1}, v_{2} \in B_{r}(X)$. The functions $L_{1}, L_{2}: \mathbb{R}_{+} \rightarrow \mathbb{R}_{+}$are non-decreasing and $B_{r}(X)$ is a closed ball in the Banach space $X$ of radius $r$.

(A3) The nonlinear operator $S: X \rightarrow X$ satisfies

$$
\|S u(\zeta)-S v(\tau)\| \leq L_{3}(r)\|u-v\|
$$

for all $\zeta, \tau \in[0, T], u, v \in B_{r}(X)$ and the function $L_{3}: \mathbb{R}_{+} \rightarrow \mathbb{R}_{+}$is non-decreasing function.

to write

Theorem 3.1. If conditions (A1) to (A3) satisfies then, the system 2.6) is trajectory controllable over the interval $[0, T]$.

Proof. Let, $y(\zeta)$ be any trajectory in $\mathcal{T}$ then we define feedback control as:

$$
\left.\left.w(\zeta)=\mathcal{D}_{0+}^{\lambda, \mu} y(\zeta)+A y(\zeta)-g(\zeta, y(\zeta), S y(\zeta))\right) d \tau\right)
$$

Putting the value of feedback control $w(\zeta)$ from equation (3.1) in equation 2.7) and simplifying we get,

$$
\mathcal{D}_{0+}^{\lambda, \mu}[u \zeta-y(\zeta)]=-A[u(\zeta)-y(\zeta)]+g(\zeta, u(\zeta), S u(\zeta))-g(\zeta, y(\zeta), S y(\zeta))
$$


Choosing $z(\zeta)=u(\zeta)-y(\zeta)$, equation 3.2 becomes:

$$
\begin{aligned}
\mathcal{D}_{0+}^{\lambda, \mu} z(\zeta) & =-A z(\zeta)+[g(\zeta, u(\zeta), S u(\zeta))-g(\zeta, y(\zeta), S y(\zeta))] \\
\mathcal{I}_{0+}^{(1-\lambda)(1-\mu)} z(0) & =0 .
\end{aligned}
$$

The mild solution of the equation $(3.3)$ is given by

$$
z(\zeta)=\int_{0}^{\zeta} \mathcal{K}_{\lambda}(\zeta-\tau)[g(\tau, u(\tau), S u(\tau))-g(\tau, y(\tau), S y(\tau))] d \tau
$$

Therefore,

$$
\begin{aligned}
\|z(\zeta)\| & \leq \int_{0}^{\zeta}\left\|\mathcal{K}_{\lambda}(\zeta-\tau)\right\|\|g(\tau, u(\tau), S u(\tau))-g(\tau, y(\tau), S y(\tau))\| d \tau \\
& \left.\leq \int_{0}^{\zeta} \frac{\tau^{\lambda-1} M}{\Gamma(\lambda)}\left[L_{1}(r)\|u(\tau)-y(\tau)\|+L_{2}(r) L_{3}(r)\right)\|u(\tau)-y(\tau)\|\right] d \tau \\
& \leq L \int_{0}^{\zeta} \tau^{\lambda-1}\|z(\tau)\| d \tau,
\end{aligned}
$$

where, $L=\frac{M\left(L_{1}(r)+L_{2}(r) L_{3}(r)\right)}{(\Gamma(\lambda))}$ and using the Gronwal's inequality we get $z(\zeta)=0$ almost everywhere. Therefore, $u(\zeta)=y(\zeta)$ almost everywhere. Hence system equation (2.6) is Trajectory controllable over the interval $[0, T]$.

Example 3.1.1. Let $X=L^{2}([0,1], \mathbb{R})$ and consider the partial differential equation

$$
\mathcal{D}_{t}^{\lambda, \mu} u(t, x)=u_{x x}(t, x)+2 u(t, x) u_{x}(t, x)+\int_{0}^{t} e^{-u(s, x)} d s+w(t)
$$

with initial condition $\mathcal{I}^{(1-\lambda)(1-\mu)} u(0, x)=u_{0}$ and boundary conditions $u(t, 0)=u(t, 1)=0$.

Define an operator $A$ as $A u=u^{\prime \prime}$ over the domain $D(A)=H^{2}(0,1) \cap H^{1}(0,1)$ then the operator $A$ is generates infinitesimal generator of $C-0$ strongly continuous semigroup $\mathcal{T}(\zeta)$ given by

$$
\mathcal{T}(\zeta)=\sum_{n=1}^{\infty} \exp \left(-n^{2} \pi^{2} \zeta\right)<u, \phi_{n}>\phi_{n}
$$

where, $\phi_{n}$ are orthonormal Fourier basis for $X$.

The equation (3.5) can be rewritten as the abstract equation in $\left.X=L^{(}[0,1], \mathbb{R}\right)$ as:

$$
\begin{aligned}
\mathcal{D}_{t}^{\lambda, \mu} u(\zeta) & =A u(\zeta)+g(\zeta, u(\zeta), S u(\zeta))+w(\zeta) \\
\mathcal{I}^{(1-\lambda)(1-\mu)} u(0) & =u_{0}
\end{aligned}
$$

Clearly, $g(\zeta, u(\zeta), S u(\zeta))=2 u u_{x}+\int_{0}^{\zeta} e^{-} u(\tau) d \tau$ is smooth function therefore for there exist $L_{1}, L 2$ and $L_{3}$ such that $\|g(\zeta, u(\zeta), S u(\zeta))-g(\zeta, v(\zeta), S v(\zeta))\|\left[\leq L_{1}(r)+L_{2}(r) L_{3}(r)\right]\|u-v\|$ for all $u, v \in B(X)$. Hence, by Theorem 3.1. the system (3.5) is trajectory controllable over the interval $[0,1]$. 


\section{Trajectory Controllbility with Nonlocal Condition}

In this section we are going to discuss trajectory controllability of the system governed by Hilfer fractional integro-differential equation in the Banach space $X$.

$$
\begin{aligned}
\mathcal{D}_{0+}^{\lambda, \mu} u(\zeta)+A u(\zeta) & =g\left(\zeta, u(\zeta), \int_{0}^{\zeta} a(\zeta, \tau, u(\tau)) d \tau\right)+w(\zeta) \\
\mathcal{I}_{0+}^{(1-\lambda)(1-\mu)}[u(0)-h(u)] & =u_{0},
\end{aligned}
$$

where, $\mathcal{D}_{0+}^{\lambda, \mu}$ is Hilfer fractional derivative operator. $A$ is closed linear operator which is infinitesimal generator of $C_{0}$-semigroup and $u_{0} \in X . w \in U$, a Hilbert space.

Definition 4.1. A function $u \in \mathcal{C}_{\mathcal{T}}$ is called mild solution of integro-differential equation (4.1) if $u$ is solution of the integral equation

$$
u(\zeta)=\mathcal{S}_{\lambda, \mu}\left[u_{0}+\mathcal{I}_{0+}^{(1-\lambda)(1-\mu)} h(u)\right]+\int_{0}^{\zeta} \mathcal{K}_{\lambda}(\zeta-\tau)[g(\tau, u(\tau), S u(\tau))+w(\tau)] d \tau
$$

where, $S u(\zeta)=\int_{0}^{\zeta} a(\zeta, \tau, u(\tau)) d \tau$, operators $\mathcal{S}_{\lambda, \mu}$ and $\mathcal{K}_{\lambda}(\zeta)$ are defined as 2.4 and 2.5 respectively.

To discuss the trajectory controllability of the system 4.1) we required following condition on $h$.

(A4) The function $h$ satisfies

$$
\|h(u)-h(v)\| \leq L_{h}\|u-v\|
$$

for all $u, v \in C([0, T], X)$.

Theorem 4.1. If conditions $(A 1)$ to $(A 4)$ satisfies then, the system 4.1) is trajectory controllable over the interval $[0, T]$ provided $L^{*} \neq 1$.

Proof. Let, $x(\zeta)$ any trajectory in $\mathcal{T}$ then define

$$
\left.\left.w(\zeta)=\mathcal{D}_{0+}^{\lambda, \mu} x(\zeta)+A x(\zeta)-g(\zeta, x(\zeta), S x(\zeta))\right) d \tau\right)
$$

Plugging the value of $w(\zeta)$ in equation 4.1) from equation (4.3) and simplifying we get,

$$
\mathcal{D}_{0+}^{\lambda, \mu}[u(\zeta)-x(\zeta)]=-A[u(\zeta)-x(\zeta)]+g(\zeta, u(\zeta), S u(\zeta))-g(\zeta, x(\zeta), S x(\zeta))
$$

Choosing $z(\zeta)=u(\zeta)-y(\zeta)$, equation 4.4 becomes:

$$
\begin{aligned}
\mathcal{D}_{0+}^{\lambda, \mu} z(\zeta) & =-A z(\zeta)+[g(\zeta, u(\zeta), S u(\zeta))-g(\zeta, x(\zeta), S x(\zeta))] \\
\mathcal{I}_{0+}^{(1-\lambda)(1-\mu)}[u(0)-x(0)-h(u)+h(x)] & =0
\end{aligned}
$$

The mild solution of the equation 4.5 is given by

$$
z(\zeta)=\mathcal{S}_{\lambda, \mu} I^{(1-\lambda)(1-\mu)}[h(u)-h(x)]+\int_{0}^{\zeta} \mathcal{K}_{\lambda}(\zeta-\tau)[g(\tau, u(\tau), S u(\tau))-g(\tau, x(\tau), S x(\tau))] d \tau
$$


Therefore,

$$
\begin{aligned}
\|z(\zeta)\| & \leq\left\|\mathcal{S}_{\lambda, \mu} I^{(1-\lambda)(1-\mu)}[h(u)-h(x)]\right\|+\int_{0}^{\zeta}\left\|\mathcal{K}_{\lambda}(\zeta-\tau)\right\|\|g(\tau, u(\tau), S u(\tau))-g(\tau, x(\tau), S x(\tau))\| d \tau \\
& \left.\leq L^{*}\|u(\zeta)-x(\zeta)\|+\int_{0}^{\zeta} \frac{\tau^{\lambda-1} M}{\Gamma(\lambda)}\left[L_{1}(r)\|u(\tau)-y(\tau)\|+L_{2}(r) L_{3}(r)\right)\|u(\tau)-y(\tau)\|\right] d \tau \\
& \leq L^{*}\|z(\zeta)\|+L \int_{0}^{\zeta} \tau^{\lambda-1}\|z(\tau)\| d \tau \\
& \leq \frac{L}{1-L^{*}} \int_{0}^{\zeta} \tau^{\lambda-1}\|z(\tau)\| d \tau
\end{aligned}
$$

where, $L=\frac{M\left(L_{1}(r)+L_{2}(r) L_{3}(r)\right)}{(\Gamma(\lambda))}$ and $L^{*}=\frac{M L_{h} T}{\Gamma(\lambda) \Gamma(\mu(1-\lambda)) \Gamma(1-\lambda)(1-\mu)+1)}$ and using Gronwall's inequality get $z(\zeta)=0$ almost everywhere. Hence, system (4.1) is trajectory controllable over $[0, T]$.

Example 4.1.1. Let $X=L^{2}([0,1], \mathbb{R})$ and consider the partial differential equation

$$
\mathcal{D}_{t}^{\lambda, \mu} u(t, x)=u_{x x}(t, x)+2 u(t, x) u_{x}(t, x)+\int_{0}^{t} e^{-u(s, x)} d s+w(t)
$$

with initial condition $\mathcal{I}^{(1-\lambda)(1-\mu)}[u(0, x)+h(u])=u_{0}, h(u)=\sum_{i=1}^{2} \frac{1}{3^{i}} u(1 / i, x)$ and boundary conditions $u(t, 0)=u(t, 1)=0$. The equation 4.7) is converted into abstract equation

$$
\begin{aligned}
\mathcal{D}_{t}^{\lambda, \mu} u(\zeta) & =A u(\zeta)+g(\zeta, u(\zeta), S u(\zeta))+w(\zeta) \\
\mathcal{I}^{(1-\lambda)(1-\mu)}[u(0)-h(u)] & =u_{0}
\end{aligned}
$$

Since $h$ is Lipchitz continuous with Lipschitz constant $L_{h}$, the system 4.7 is trajectory controllable over the interval $[0,1]$.

\section{Conclusion}

In this manuscript we have discussed sufficient conditions for the trajectory controllability of system governed by integro-differential systems with local and nonlocal conditions on general Banach space. We have also added illustratioins to validate the derived results.

\section{References}

[1] Barenblat, G., Zheltor, J., Kochiva, I. (1960). Basic concepts in the theory of seepage of homogeneous liquids in fissured rocks. J. Appl. Math. Mech. 24:1286-1303. DOI: 10.1016/00218928(60)90107-6.

[2] Hilfer, R. (2000). Applications of Fractional Calculus in Physics. Singapore: World Scientific. 
[3] Furati, K. M., Kassim, M. D., Tatar, N. E. (2012). Existence and uniqueness for a problem involving hilfer fractional derivative. Comput. Math. Appl. 641:616-626.

[4] Debbouche, A., Antonov, V. (2017). Approximate controllability of semilinear Hilfer fractional differential inclusions with impulsive control inclusion conditions in Banach spaces. Chaos Solitons Fractals 102:140-148. DOI: 10.1016/ j.chaos.2017.03.023.

[5] Gu, H., Trujillo, J. J. (2015). Existence of integral solution for evolution equation with Hilfer fractional derivative. Appl. Math. Comput. 257:344-354. DOI: 10.1016/j.amc.2014.10.083.

[6] Jin, R. W., Ahmed, G. I., O'Regan, D. (2018). Hilfer-type fractional differential switched inclusions with noninstantaneous impulsive and nonlocal conditions. Nonlinear Anal. Model. Control 23:921-941. DOI: 10.15388/NA.2018.6.7.

[7] Wang, J. R., Zhang, Y. (2015). Nonlocal initial value problems for differential equations with hilfer fractional derivative. Appl. Math. Comput. 266:850-859. DOI: 10.1016/j.amc.2015.05.144.

[8] Yang, M., Wang, Q. R. (2017). Approximate controllability of hilfer fractional differential inclusions with nonlocal conditions. Math. Meth. Appl. Sci. 40(4):1126-1138. DOI: 10.1002/mma.4040.

[9] Kilbas, A. A., Srivastava, H. M., Trujillo, J. J. (2006). Theory and Applications of Fractional Differential Equations. North-Holland Mathematics Studies 204. Amsterdam, the Netherlands: Elsevier Science B.V.

[10] Bashirov, A.E., Mahmudov, N.I.: On concepts of controllability for deterministic and stochastic systems, SIAM J. Control Optim. 37, 1808-1821 (1999)

[11] Benchohra, M., Gatsori, E.P., Ntouyas, S.K.: Controllability results for semilinear evolution inclusion with nonlocal conditions, J. Optim. Theory Appl. 118(3), 493-513 (2003)

[12] Cindea, N., Tucsnak, M.: Local exact controllability for Berger plate equation, Math. Control Signals Syst. 21, 93-110 (2009)

[13] Chalishajar, D.N.: Controllability of second order impulsive neutral functional differential inclusions with infinite delay, J. Optim. Theory Appl. 154(2), 672-684 (2012)

[14] Chalishajar, D.N.: Controllability of mixedVolterra-Fredholm-type integro-differential systems inBanach space. J. Frankl. Inst. 344, 12-21 (2007)

[15] Jafari, H., Tajadodi, H.: Fractional order optimal control problems via the operational matrices of berstein polynomials, U. P. B. Sci. Bull. Ser. A 76(3), 115-128 (2014)

[16] Muslim,M., Agarwal, R.P.,Mahmudov, N.I.: Approximate controllability of integro-differential equations in a Hilbert space with nonlocal conditions. Dyn. Contin, Discrete Impuls. Syst. Ser. A Math. Anal. 18, 269-283 (2011) 
[17] Mahmudov, N.I.: Approximate controllability of semilinear evolution systems in Hilbert spaces, SIAM.J. Control Optim. 15(1977), 407-411 (1980). Addendum: 18: 98-99

[18] Mahmudov, N.I.: Approximate controllability of evolution systems with nonlocal conditions, Nonlinear Anal. Theory Methods Appl. 68(3), 536-546 (2008)

[19] Mahmudov, N.I., Mckibben, M.A.: Approximate controllability of second order neutral stochastic evolution equations, Dyn. Contin. Discrete Impuls. Syst. 13, 619-634 (2006)

[20] Sakthivel, R.,Mahmudov, N.I., Nieto, J.J.: Controllability for a class of fractional-order neutral evolution control systems, Appl. Math. Comput. 218, 10334-10340 (2012)

[21] Sakthivel, R., Anthoni, S.M., Kim, J.H.: Existence and controllability result for semi-linear evolution integrodifferential systems, Math. Comput. Model. 41, 1005-1011 (2005)

[22] Singh V., Controllability of Hilfer Fractional Differential Systems with Non-Dense Domain, Numerical Funictional Analysis and Optimization, 40(13) 2019, 1572-1592

[23] George, R. K., Trajectory controllability of 1-dimensional nonlinear systems. In Proceedings of the Research Seminar in honour of Professor M.N. Vasavada (pp. 43-48). Anand, India: S.P. University.

[24] Chalishajar, D.N., George, R.K., Nandakumaran, A.K., Acharya, F.S.: Trajectory controllability of nonlinear integro-differential system, J. Frankl. Inst. 347(7), 1065-1075 (2010)

[25] Govindaraj, V., Muslim, M. and George, R. K., Trajectory controllability of fractional dynamical systems, Journal of Control and Decision, 4(2), 114-130 (2017)

[26] Dhayal, R., Muslim, M., Abbas, S., Approximate and Trajectory controllability of Fractional Neutral Differential Equation, Adv. Oper. Theory 4 (2019), no. 4, 802-820

[27] Muslim, M., George, R. K., Trajectory Controllability of the Nonlinear Systems Governed by Fractional Differential Equations, Differ Equ Dyn Syst (October 2019) 27(4), 529-537

[28] Jaiswal, A., Bahuguna, D., Hilfer fractional Differential Equations with Almost Sectorial Operators, Differ Equ Dyn Syst (2020). https://doi.org/10.1007/s12591-020-00514-y 